\title{
Influence of hiring, selection process and safety training on lightning safety performance in Iranian manufacturing companies
}

\begin{abstract}
There are a high number of studies in the area of safety performance in organizational level, but scarce research exists in lightning safety performance and its antecedents. This study has identified factors of safety training and hiring and selection process which are hypothesized to enhance lightning safety performance and examined the degree to which they have contribution in lightning safety performance in Iranian companies. Results confirmed that the predictors of hiring and selection process and safety training are positively contributed to lightning safety performance in Iranian manufacturing companies.
\end{abstract}

Keyword: Hiring and selection; Safety training; Lightning safety performance 